\title{
Large quadrature squeezing at high intensities
}

\author{
C. Cabrillo and F. J. Bermejo \\ Instituto de Estructura de la Materia, Consejo Superior de Investigaciones Cientificas, Serrano 123, 28006 Madrid, Spain
}

(Received 11 March 1993)

\begin{abstract}
The squeezing properties of a system characterized by large $\chi^{(2)}$ and $\chi^{(3)}$ nonlinear susceptibilities and injected classical fields for the two relevant modes are studied as a function of the output power. A large reduction of noise levels well above threshold has been found and a remarkable dependence of the maximum squeezing on the input power has been shown. The possibility of an implementation with state of the art technology is demonstrated, enabling a practical realization of a laserlike source with a high degree of squeezing at high intensities.
\end{abstract}

PACS number(s): 42.50.Dv, 42.65.Ky

Despite the wide range of possible applications of quadrature-squeezed light $[1,2]$ opened up by the first experimental generation of these nonclassical fields [3], the advances in the production of squeezed light with usable intensities have been somewhat modest. As an example, only very recently [4], a significant output power of 0.17 $\mathrm{mW}$ was achieved for a beam which, on the other hand, was shown to exhibit a small noise reduction, leading to a degree of squeezing of about $18 \%$. Leaving aside the experimental problems associated with the operation of sources for bright squeezing well above threshold, the underlying reasons for this difficulty can be found even at a theoretical or model-building level. As a matter of fact, most of the reported models predict a strong degradation of squeezing when the input power is raised at the levels required to generate a bright output. Such a difficulty can be circumvented by the introduction of an injected signal, something found to be useful for counterbalancing the deleterious effect caused by high input powers [5].

In the search for a suitable model device, an optical parametric oscillator (OPO) with an added Kerr-effect term was considered previously $[6,7]$. As a result it was found that, although the maximum available squeezing was improved in comparison with the standard OPO, very low efficiency in the down-conversion process resulted [7]. To compensate for such a decrease, consideration was made of arguments given by Tombesi [8], where an injected classical signal was shown to lead to a substantial reduction of the interaction time needed to achieve strong noise reduction.

As it will be shown below, such a model device enables the production of bright-squeezed light, showing a remarkable dependence on the output power. The relevance of this study goes well beyond the theoretical study of such a device since, as it will be illustrated below, recent advances in the technology of multiple quantum wells (MQW) enable its practical implementation based upon already existing devices.

To specify such a model system, two quantized modes $\hat{a}$ and $\hat{b}$ both excited by injected classical fields, with frequencies $\omega$ and $2 \omega$, respectively, are considered. Both modes are resonant in an optical ring cavity where they interact with a suitable nonlinear medium characterized by its second $\chi_{2 \omega}^{(2)}$ and third $\chi_{\omega,-\omega, \omega}^{(3)}$ order susceptibilities.
The losses in the input-output mirror are accounted for by means of independent reservoir interactions for each mode. An effective Hamiltonian for the model system can be written as $(\hbar=1)$

$$
\begin{aligned}
H= & \omega \hat{a}^{\dagger} \hat{a}+2 \omega \hat{b}^{\dagger} \hat{b}+\frac{i}{2}\left[\kappa\left(\hat{a}^{\dagger}\right)^{2} \hat{b}-\kappa^{*} \hat{a}^{2} \hat{b}^{\dagger}\right]+\frac{\Gamma}{2}\left(\hat{a}^{\dagger}\right)^{2} \hat{a}^{2} \\
& +i\left(E_{b} e^{i 2 \omega t} \hat{b}-E_{b}^{*} e^{-i 2 \omega t} \hat{b}^{\dagger}\right) \\
& +i\left(E_{a} e^{i \omega t} \hat{a}-E_{a}^{*} e^{-i \omega t} \hat{a}^{\dagger}\right) \\
& +\hat{a} \Gamma_{A}^{\dagger}+\hat{a}^{\dagger} \Gamma_{A}+\hat{b} \Gamma_{B}^{\dagger}+\hat{b}^{\dagger} \Gamma_{B}
\end{aligned}
$$

where $\kappa$ and $\Gamma$ are proportional to the $\chi_{2 \omega}^{(2)}$ and $\chi_{\omega,-\omega, \omega}^{(3)}$ susceptibilities, respectively, $E_{a}$ and $E_{b}$ are related to the complex amplitudes of the external classical fields as well as to the transmission coefficients of the inputoutput mirror corresponding respectively to the $\omega$ and $2 \omega$ modes, and $\Gamma_{A}$ and $\Gamma_{B}$ are the usual reservoir operators. Following the same lines drawn in Ref. [7] a couple of stochastic differential equations are obtained by means of a mapping onto phase space using a $P$ representation. The arbitrary phases and the oscillatory part are removed by passing to a new frame of reference,

$$
\begin{aligned}
& \hat{\alpha}=\sqrt{\frac{|\kappa|^{2}}{2 \gamma_{a} \gamma_{b}}} \exp \left\{-i\left[\left(\theta_{k}+\theta_{b}\right) / 2-\omega t\right]\right\} \hat{a}, \\
& \hat{\beta}=\frac{|\kappa|}{2 \gamma_{a}} \exp \left\{-i\left[\theta_{b}-2 \omega t\right]\right\} \hat{b},
\end{aligned}
$$

with $\gamma_{a}$ and $\gamma_{b}$ the loss rates through the input-output mirror for the $\omega, 2 \omega$ modes, and $\theta_{\kappa}$ and $\theta_{b}$ the phases of $\kappa$ and $E_{b}$, respectively. The deterministic part of the stochastic equations then takes the form

$$
\begin{aligned}
& \frac{d \alpha}{d \tau}=-\alpha+\beta \alpha^{*}-i \Lambda \alpha^{*} \alpha^{2}+\sqrt{\frac{r}{2}} \lambda_{a}, \\
& \frac{d \beta}{d \tau}=-r \beta-r \alpha^{2}+r \lambda_{b},
\end{aligned}
$$

where

$$
\tau=\gamma_{a} t, r=\frac{\gamma_{b}}{\gamma_{a}}, \Lambda=\frac{2 \gamma_{b} \Gamma}{|\kappa|^{2}}
$$


$\lambda_{a}=\frac{|\kappa|\left|E_{a}\right|}{\gamma_{a} \gamma_{b}} \exp \left\{i\left[\theta_{a}-\left(\theta_{k}+\theta_{b}\right) / 2\right]\right\}, \quad \lambda_{b}=\frac{|\kappa|\left|E_{b}\right|}{\gamma_{a} \gamma_{b}}$

$\theta_{a}$ is the phase of $E_{a}$, and $\alpha$ and $\beta$ are the complex amplitudes associated with the quantum operators $\hat{\alpha}$ and $\hat{\beta}$. It is worth noting that the independent term of the first equation retains an irreducible phase, and all the other parameters involved in the problem are now positive real numbers (including the zero). The following relation between the phase and modulus of $\alpha_{f}$ (the fixed points of mode $\omega$ ) results after equating to zero the left-hand side of Eq. 1,

$$
\tan \varphi_{f}=-\frac{\Lambda\left|\alpha_{f}\right|^{2}-\tan \left(\varphi_{a}\right)\left(\left|\alpha_{f}\right|^{2}-\lambda_{b}+1\right)}{\tan \left(\varphi_{a}\right) \Lambda\left|\alpha_{f}\right|^{2}+\left(\left|\alpha_{f}\right|^{2}+\lambda_{b}+1\right)}
$$

$\varphi_{a}$ being the phase of $\lambda_{a}$. This relation holds for any value of $\varphi_{a}$ within $[0,2 \pi)$, so that values of $\varphi_{f}$ within $[0,2 \pi)$ are allowed. Note that the absence of an injected classical signal for mode $\omega$ (i.e., $\lambda_{a}=0$ ) restricts this interval to $[7 \pi / 4,2 \pi][7]$. At the same time, once $\left|\alpha_{f}\right|^{2}$ and $\varphi_{f}$ are given, $\lambda_{a}$ and $\varphi_{a}$ are fixed.

Following procedures described in Ref. [9], a set of equations which are formally the same than those given in our previous work [7] is found, since only an independent term has been added to the differential equations. The explicit form for the eigenvalues also follows as [7]

$$
\begin{aligned}
& k_{1}, k_{2}=-\frac{1}{2}(r+1-g) \pm \frac{1}{2}\left[(-r+1-g)^{2}-8 r\left|\alpha_{f}\right|^{2}\right]^{1 / 2}, \\
& k_{3}, k_{4}=-\frac{1}{2}(r+1+g) \pm \frac{1}{2}\left[(-r+1+g)^{2}-8 r\left|\alpha_{f}\right|^{2}\right]^{1 / 2},
\end{aligned}
$$

where $g$ is now given by

$$
g=+\sqrt{\left|\lambda_{b}-\alpha_{f}^{2}(1+i \Lambda)\right|^{2}-4 \Lambda^{2}\left|\alpha_{f}\right|^{4}} .
$$

The case considered in our previous study [7] (without injected signal) is easily recovered by setting $\lambda_{a}=0$ and $\lambda_{b} \geq 1$ which leads to $\left|\lambda_{b}-\alpha_{f}^{2}(1+i \Lambda)\right|^{2}=1$.

The stability analysis evidences a Hopf bifurcation frontier [10] at $g=r+1$ where $\operatorname{Re} k_{1}=\operatorname{Re} k_{2}=0 \neq$ $\operatorname{Im} k_{1}=-\operatorname{Im} k_{2}$ which gives rise to self-sustained oscillations when $g>r+1$ and a saddle-node or pitchfork bifurcation frontier [10] at $g=1+2\left|\alpha_{f}\right|^{2}$ where $k_{2}=0$. A set of stable fixed points $\alpha_{s}$ is then found for $g<r+1$ and $g<1+2\left|\alpha_{s}\right|^{2}$.

The spectrum of squeezing is then calculated following Collet and Gardiner $[11,12]$, where perfect squeezing occurs at -0.25 . If the field quadratures are defined as $\hat{a}(t)=e^{-i \theta}[\hat{x}(t, \theta)+i \hat{y}(t, \theta)]$ the spectrum of squeezing is given within the conditions of validity of the input-output formalism $[11,12]$

$$
\begin{aligned}
& : S(\omega, \theta)^{\text {out }}: \\
& \quad=2 \gamma_{a} \int_{-\infty}^{\infty}\langle[\hat{x}(t, \theta)-\langle\hat{x}\rangle][\hat{x}(t+\tau, \theta)-\langle\hat{x}\rangle]\rangle e^{i \omega \tau} d \tau,
\end{aligned}
$$

where \langle\rangle denotes a quantum average.

An analytical minimization of : $S(\omega, \theta)^{\text {out }}:$ in $\theta$ for each $\omega$ was carried out. For cases where $\Lambda \neq 0$ (i.e., higher order effects are operative) the optimal value of $\theta$ depends on $\omega$, so that the resulting spectrum : $S(\omega)^{\text {out }}$ : does not correspond to any measure along a given direction on the phase space, but to obtain one point of our squeezing spectrum the noise power at a given frequency should be measured (using high resolution in energy) scanning the phase of the local oscillator, seeking for the minimum spectral variance (see, for instance, Ref. [4] for a measure of bright squeezing at $150.5 \mathrm{Mhz}$ ). Also, for given values of $\left|\alpha_{s}\right|^{2}, \lambda_{b}, \Lambda$, and $r,: S(\omega)^{\text {out }}$ : has been numerically minimized in the frequency and phase of $\alpha_{s}$. Such a procedure provides the maximum available squeezing, : $S_{m}^{\text {out }}$ : as a function of $\left|\alpha_{s}\right|^{2}$, and it has been applied to both modes $\omega$ and $2 \omega$.

Figure 1 exemplifies the influence of $\Lambda$ on $: S_{m}^{\text {out }}:$ for mode $\omega$, where a dramatic improvement of squeezing is found. The gap between the origin of coordinates and the beginning of the curves corresponds to the instability region before the saddle-node point. The end of the curve marked $a$ (pure sub-second-harmonic generation) is located at the Hopf bifurcation. The presence of this bifurcation is very sensitive to a nonzero value of $\Lambda$ and is not present in the other cases shown. Maximum squeezing appears for higher photon numbers as $\Lambda$ is increased up to $1 . \Lambda=1$ (i.e., in effective terms the third and second order effects become comparable) seems to be a limiting case for which the maximum is located at $\infty$, but it comes back to lower photon numbers and disappears for high enough values of $\Lambda$. To our knowledge this is the first time that a nonmonotonic decreasing dependence with respect to the output power is observed. To get a large degree of bright quadrature squeezing with the model systems proposed up to now, the working point must be located near an instability, where perfect squeezing can be reached, as an increase of input power quickly degrades the squeezing. However, for practical realizations the working points must be far from instabilities. The very remarkable effect of quantum noise reduction with increasing power shown here opens the possibility for the practical realization of a bright squeezer. The case $\Lambda=1$ merits a special consideration since it shows

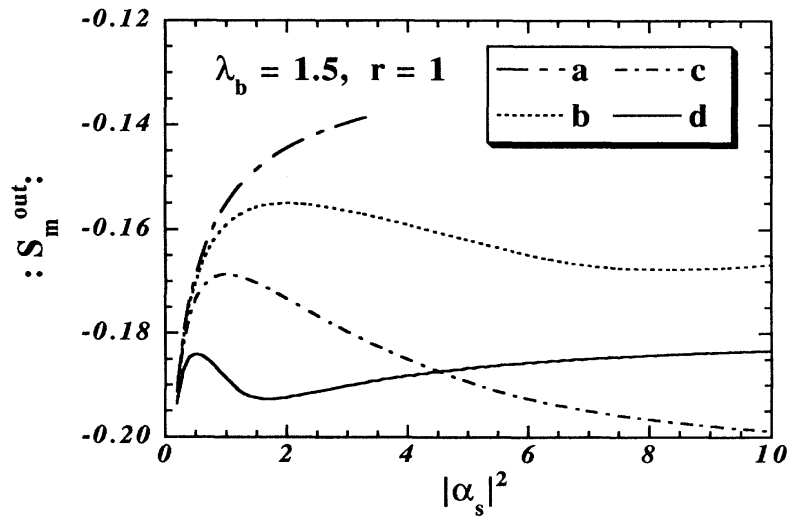

FIG. 1. The maximum available squeezing as a function of the photon number for four different cases: curve $a, \Lambda=0$; curve $b, \Lambda=0.5$; curve $c, \Lambda=1$; curve $d, \Lambda=2$. In all cases $\lambda_{b}=1.5$ and $r=1$. 
a residual squeezing (i.e., at very large output power) around $83 \%$ which represents an improvement of $86 \%$ with respect to the residual squeezing of the conventional OPO. Large noise reduction is also obtained for mode $2 \omega$, although less than that achieved for the other mode. It is worth remarking that the system presents a large flexibility enabling large degrees of squeezing at almost any output power.

A comparison of the present results with those of subharmonic generation (the standard OPO), secondharmonic generation, and sub-second-harmonic generation, with parameters chosen to reach the maximum at moderate output power (with the normalization used, about 0.6 times the input power of the OPO at threshold), is shown in Fig. 2(a). All the curves end at the Hopf bifurcation of second-harmonic generation. In the cases shown in Fig. 1 the increase in output power is a consequence of higher input power of mode $\omega$ as $\lambda_{b}$ is kept constant (1.5). In counterposition, curve $e$ now corresponds to $\lambda_{b}=4$ with $\Lambda$ chosen to reach the maximum
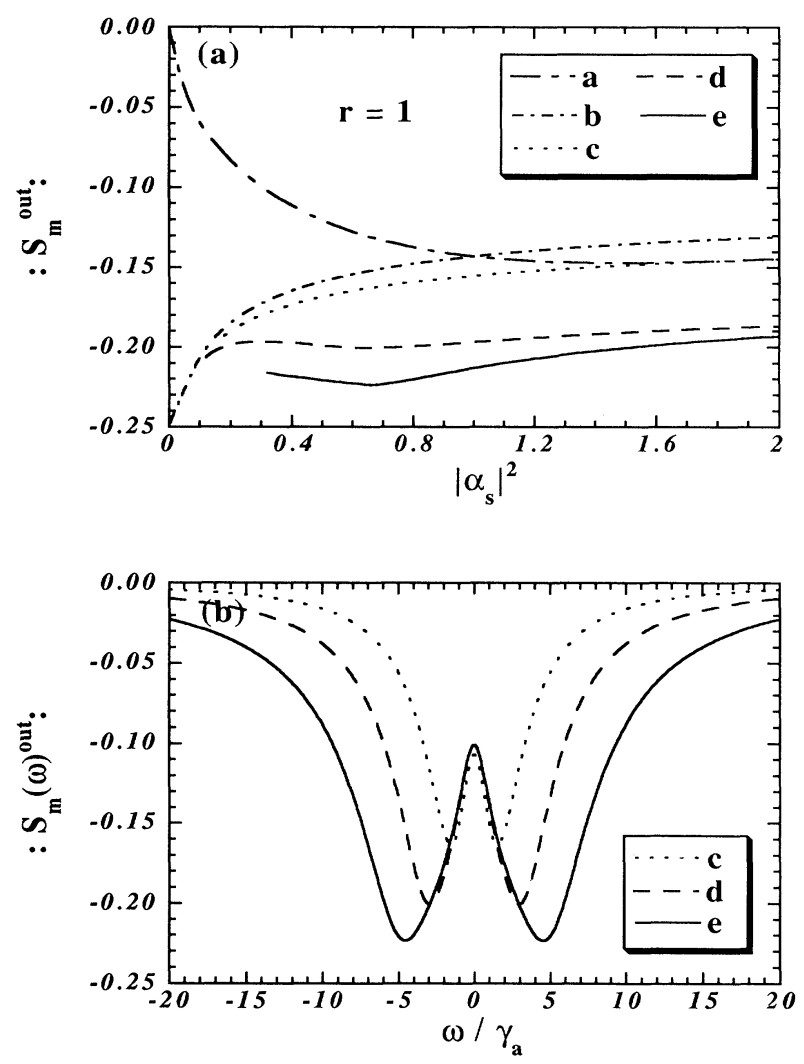

FIG. 2. The maximum available squeezing as a function of the photon number for five different cases (a) and the corresponding spectrum at $\left|\alpha_{s}\right|^{2}=0.64$ for the three most favorable cases (b): curve $a$, second-harmonic generation $\left(\lambda_{b}=1.5\right)$; curve $b$, subharmonic generation $\left(\lambda_{b}=1.5\right)$; curve $c$, sub-second-harmonic generation $\left(\lambda_{b}=1.5\right)$; curve $d, \Lambda=3.5\left(\lambda_{b}=1.5\right)$; curve $e, \Lambda=7.5\left(\lambda_{b}=4\right)$. In all cases $r=1$. at the same photon number, and as can be easily seen, the rise in input power increases the reduction of quantum noise. Furthermore, the variance of the unsqueezed quadrature is also reduced. A strikingly large squeezing of $90 \%$ is reached in this case. Figure 2(b) shows the optimum output spectrum, $: S_{m}(\omega)^{\text {out }}:$, of the three most favorable cases of Fig. 1(a) at $\left|\alpha_{s}\right|^{2}=0.64$. As it is clear from the figure the best case implies an improvement of around $37 \%$ with respect to the sub-second-harmonic generation, and, furthermore, the spectrum now covers a wider extent in frequencies. We have scanned a wide range of parameters and a large enhancement of squeezing has been obtained in all cases.

Although the practical implementation of such a system requires large values of the $\chi_{2 \omega}^{(2)}$ and $\chi_{\omega,-\omega, \omega}^{(3)}$ susceptibilities, recent advances in multiple quantum wells have enabled the construction of devices showing huge resonant nonlinear susceptibilities $[13,14]$. In particular, the doubly resonant case of [13] is an ideal device since both $\chi_{2 \omega}^{(2)}$ and $\chi_{\omega,-\omega, \omega}^{(3)}$ susceptibilities are largely enhanced. Although, to the authors' knowledge, no measurements of $\left|\chi_{\omega,-\omega, \omega}^{(3)}\right|$ have been made, some estimations can be made using for the purpose the same kind of approximation as employed for the calculation of $\chi_{2 \omega}^{(2)}$. As a result, we have estimated $\left|\chi_{\omega,-\omega, \omega}^{(3)}\right| \simeq 1.4 \times 10^{-13}(\mathrm{~m} / \mathrm{V})^{2}$ at resonance $(122.2 \mathrm{meV})$. Using the experimental value of $\left|\chi_{2 \omega}^{(2)}\right|\left(7.5 \times 10^{-8} \mathrm{~m} / \mathrm{V}\right)$ and setting $\Lambda=3.5, r=1$, and a cavity length of $1 \mathrm{~cm}$ we obtain $\gamma_{a} \simeq 2 \times 10^{8} \mathrm{~s}^{-1}$. This implies a reflectivity of $R \simeq 99 \%$, something which allows the use of the input-output theory. The effective interaction length is $\simeq 87 \mu \mathrm{m}$ with a coherence length of $\simeq 120$ $\mu \mathrm{m}$, something which obviates stringent phase matching conditions. The corresponding parameter $\gamma$ for the absorption measured in the material is five orders of magnitude less than $\gamma_{a}$ and could be neglected. For $\lambda_{b}=1.5$ which corresponds to case $d$ of Fig. 2(b) (80\% of squeezing) an input power of $\simeq 120 \mathrm{~mW}$ at $2 \omega$ and $\simeq 190 \mathrm{~mW}$ at $\omega$ is needed, giving an output power of $\simeq 30 \mathrm{~mW}$ at frequency $\omega$. These power requirements are feasible with standard laser systems. It is worth emphasizing that no special requirements for the MQW are considered for this estimation so that a heterostructure specifically designed for this purpose could well improve the efficiency. On the other hand it should be noticed that due to the huge nonlinear susceptibilities involved the added fourth order term is a necessary correction for squeezing calculations in doubly resonant asymmetric MQW.

In summary, the present contribution has analyzed a model system which shows a large enhancement of squeezing with respect to systems proposed up to date and a very remarkable dependence of squeezing on the increase of input power. The large flexibility of working points and the possibility of its practical realization with state of the art MQW technology enables a realistic possibility of laserlike sources with a high degree of quadrature squeezing.

Work supported in part by Grant No. TIC-90/80 (DGICYT, Spain). 
[1] For possible applications to optical communications, see Quantum Aspects of Optical Communications, edited by C. Bendjaballah et al. (Springer-Verlag, Berlin, 1991).

[2] A review regarding a wider scope of applications can be found in Y. Yamamoto, S. Machida, S. Saito, N. Imoto, T. Yanagawa, M. Kitagawa, and G. Björk, in Progress in Optics, edited by E. Wolf (Elsevier, Amsterdam, 1990), Vol. 28, p. 89.

[3] R. E. Slusher et al., Phys. Rev. Lett. 55, 2409 (1985).

[4] D. M. Hope et al., Phys. Rev. A 46, R1181 (1992).

[5] F. A. M. de Oliveira and P. L. Knight, Phys. Rev. Lett. 61, 830 (1988).

[6] C. Cabrillo, F. J. Bermejo, P. Garcia-Fernendez, R. Toral, P. Colet, and M. San Miguel, Phys. Rev. A 45, 3216 (1992).
[7] C. Cabrillo and F. J. Bermejo, Phys. Lett. A 170, 300 (1992).

[8] P. Tombesi, Phys. Rev. A 39, 4288 (1989).

[9] M. J. Collet and D. F. Walls, Phys. Rev. A 32, 2887 (1985).

[10] J. Guckenheimer and P. Holmes, Nonlinear Oscillations, Dynamical Systems, and Bifurcations of Vector Fields (Springer-Verlag, New York, 1986).

[11] M. J. Collet and C. W. Gardiner, Phys. Rev. A 30, 1386 (1984)

[12] C. W. Gardiner and M. J. Collet, Phys. Rev. A 31, 3761 (1985).

[13] C. Sirtori et al., Appl. Phys. Lett. 60, 151 (1992).

[14] C. Sirtori et al., Phys. Rev. Lett. 68, 1010 (1992). 